\title{
Epidemiology of norovirus gastroenteritis in Germany 2001-2009: eight seasons of routine surveillance
}

\author{
H. BERNARD ${ }^{1 *}$, M. HÖHNE ${ }^{2}$, S. NIENDORF ${ }^{2}$, D. ALTMANN ${ }^{1}$ ANd K. STARK \\ ${ }^{1}$ Robert Koch Institute, Department for Infectious Disease Epidemiology, Berlin, Germany \\ ${ }^{2}$ Robert Koch Institute, Department of Infectious Diseases, Consultant Laboratory for Noroviruses, \\ Berlin, Germany
}

Received 30 July 2012; Final revision 1 February 2013; Accepted 6 February 2013; first published online 21 March 2013

\section{SUMMARY}

We analysed data on laboratory or epidemiologically confirmed cases $(n=856539)$ and on outbreaks $(n=31644)$ notified during week 31 (2001) to week 30 (2009), and performed molecular typing of specimens from 665 outbreaks. We aimed at identifying demographic and molecular characteristics to inform on potential additional approaches to prevent disease spread in the population. The mean incidence by norovirus season (week 31 in one year to week 30 in the following year) was 130 (range 19-300) cases/100000 population and was highest in persons aged $<5$ years $(430 / 100000)$ and $\geqslant 75$ years $(593 / 100000)$. The proportion hospitalized in community-acquired cases was $8-19 \%$ per season. The mean norovirus-associated mortality was $0 \cdot 05 / 100000$ per season and $0 \cdot 5 / 100000$ in the $\geqslant 75$ years age group. Most outbreaks with known setting $(75 \%)$ occurred in hospitals $(32 \%)$, nursing homes $(28 \%)$, households $(24 \%)$ and childcare facilities $(10 \%)$. GII strains dominated in the outbreak specimens. GII.4 strains were found in $82 \%$ of nursing home outbreaks, $85 \%$ of hospital outbreaks, and $33 \%$ of childcare facility and school outbreaks. Cases in younger individuals were notified earlier during the season than adult cases, and outbreaks in childcare facilities and schools preceded those in nursing/residential homes, hospitals and private households. We suggest future studies to investigate more closely potential transmission patterns between children and adults.

Key words: Infectious disease epidemiology, Norwalk agent and related viruses, notifiable infectious diseases, outbreaks, surveillance.

\section{INTRODUCTION}

Noroviruses are the most frequent cause of acute gastroenteritis in both sporadic community and outbreak cases [1,2]. The disease is usually self-limiting, of short duration and characterized by vomiting

\footnotetext{
* Author for correspondence: Dr H. Bernard, Robert Koch Institute, Department of Infectious Disease Epidemiology, Gastrointestinal Infections, Zoonoses and Tropical Infections Unit, DGZ-Ring 1, 13086 Berlin, Germany. (Email: BernardH@rki.de)
}

and diarrhoea, but symptoms can persist longer in vulnerable populations such as the elderly [3] or immunocompromised [4]. Large outbreaks often occur in community facilities such as hospitals, nursing homes for the elderly, and childcare facilities [5]. Due to a high genetic diversity, noroviruses segregate into five genogroups. The three human pathogenic genogroups (GI, GII, GIV) are further divided into at least 30 different genotypes [6]. The majority of worldwide epidemics have been caused by drift variants of genotype II.4 [7, 8]. 
In Germany, norovirus gastroenteritis is a notifiable illness according to the Protection Against Infection Act of 2001 and nationwide surveillance data are available in an electronic database at the Robert Koch Institute (RKI), which is the public health institute at the national level.

Here, we describe eight seasons of norovirus surveillance in Germany in order to identify demographic and molecular characteristics to inform on potential additional approaches to prevent disease spread in the population.

\section{METHODS}

\section{Routine surveillance data}

In Germany, laboratory detection of norovirus infection as well as cases of suspected infectious gastroenteritis or food poisoning, if occurring in food handlers or in outbreaks, are notifiable to the local public health departments (LPHD). Not only are health professionals required to report cases but also the heads of institutional facilities (e.g. residential homes, nurseries), pilots, ship captains, etc.

For every reported person meeting the surveillance case definition (see below), the LPHD completes an electronic case report which is transmitted (without the name and address of the patient) to the RKI via one of the 16 state health departments. Cases occurring within an outbreak can be linked in an electronic outbreak report at the local, the federal state or the national level of the public health system. The outbreak report contains details on the outbreak (e.g. setting, suspected vehicle of infection, evidence for suspected vehicle) and is integrated into the electronic reporting system.

In the analysis we included cases of norovirus gastroenteritis if they met the national surveillance case definition [9] which comprises cases with gastroenteritis symptoms (vomiting or diarrhoea, i.e. $\geqslant 3$ loose stools within $24 \mathrm{~h}$, or as diagnosed by the patient or healthcare personnel) with laboratory or epidemiological confirmation. Laboratory confirmation is defined as a positive result of either PCR, ELISA (from stools only) or electron microscopy. A case is considered epidemiologically confirmed by an epidemiological link to a laboratory-confirmed case through person-to-person transmission or through a common exposure (e.g. food). Alternatively, the reported consumption of a laboratory-confirmed norovirus-contaminated product also allows the establishment of an epidemiological link. We describe all reported outbreaks with at least two cases meeting the case definition and with at least one laboratoryconfirmed case.

Notification data on cases and outbreaks were extracted from the national surveillance database as of 1 January 2011. We included all cases reported between week 31 (2001) and week 30 (2009) in our analysis. After this notification period, information on cases that are only epidemiologically confirmed is no longer forwarded from the LPHD to the RKI and a comparison between seasons based on all cases can no longer be made. The incidence of reported norovirus gastroenteritis by season was calculated using the information on the German population size provided by the statistical offices of the federal states. We defined the start of an outbreak as the week the first case in the outbreak was reported. Since norovirus activity peaks during winter, a season was considered to range from week 31 in one year to week 30 in the following year. We considered cases with disease onset $\geqslant 1$ day after hospital admission as potentially nosocomial cases.

\section{Laboratory data}

For genotyping of noroviruses circulating in Germany, we analysed stool specimens from norovirus-positive outbreaks sent to the Consultant Laboratory for Noroviruses (CL) by diagnostic laboratories, physicians and local public health authorities at their convenience. For sequencing, PCR products of region $\mathrm{A}$ in the RNA-dependent RNA polymerase gene (ORF1) and region $\mathrm{C}$ in the $5^{\prime}$ terminal part of the capsid gene (ORF2) were used [10]. Sample processing, amplification, and sequencing of region A were performed as described previously $[11,12]$. For amplification and sequencing of region C, primers specific for GI and GII were used (Table 1). Amplicons were sequenced directly using the BigDye terminator cycle sequencing kit and an ABI 3130xI Genetic Analyzer (Applied Biosystems, USA). Sequences were aligned to prototype sequences drawn from GenBank with use of CLUSTAL W, version 1.6, and phylogenetic trees were produced using the neighbour-joining and DNADIST program of the Phylogeny interference package (PHYLIP), version $3.57 \mathrm{c}$ [13].

All statistical analyses were performed with Stata v. 12 (StataCorp, USA). 
Table 1. Primers used for amplification and sequencing of genogroup ( $G$ ) I and GII, and of region C (capsid gene fragment)

\begin{tabular}{llll}
\hline \hline Genogroup & Round & Primer & Sequence $\left(5^{\prime}-3^{\prime}\right)$ \\
\hline GI & First & NV192 (s) & GCYATGTTCCGCTGGATGC \\
& & NV285 (as) & CCAACCCARCCATTRTACAT \\
& Second & NV192 (s) & GCYATGTTCCGCTGGATGC \\
& & NV300/I (as) & TGRGGIGCYTGIACAAAATT \\
GII & First & NV107c (s) & AICCIATGTTYAGITGGATG \\
& & NV107c (s) & ACCKGCATAACCATTRTACAT \\
& Second & NV300/II (as) & AICCIATGTTYAGITGGATG \\
& & CYAGGKGCYTGIACAAARTT \\
\hline
\end{tabular}

I, Inosin; $\mathrm{Y}=\mathrm{C} / \mathrm{T} ; \mathrm{R}=\mathrm{A} / \mathrm{G} ; \mathrm{K}=\mathrm{G} / \mathrm{T}$.

\section{RESULTS}

\section{Time trend}

From season 2001/2002 to season 2008/2009, a total of 856539 norovirus cases were reported in Germany. The case number by season varied between 15414 in 2001/2002 and 246800 in 2007/2008, corresponding to a mean incidence per season of 130 (range 19-300) cases/100000 population (Table 2). Notification data show a strong, single-peak seasonality with the majority ( $76 \%$ ) of cases occurring between November and March. Case numbers increased over the seasons and remained at a high level since 2006/2007 (Fig. 1).

On average, $49 \%$ of cases were laboratoryconfirmed ranging from $31 \%$ in $2002 / 2003$ to $58 \%$ in $2008 / 2009$. Of the cases with laboratory confirmation, $66 \%$ were laboratory-confirmed only, whereas $34 \%$ were also epidemiologically confirmed. The proportion of laboratory-confirmed cases differed within each season. It was high during the months with lower incidences at the end of each season (July: $65 \%$ ) and low during the high season (December: $41 \%)$.

Seventy-six percent of cases were reported in outbreaks ranging from $56 \%$ in $2003 / 2004$ to $87 \%$ in 2001/2002 (Table 2, Fig. 1), with variations between summer (July: 43\%) and winter (November-January: $74 \%)$.

Cases in children aged $0-5$ years and in pupils aged 6-17 years were notified earlier during the season than cases in socially active adults (18-74 years) and the socially less active elderly ( $\geqslant 75$ years, Fig. 2$)$. In the two youngest age groups, $25 \%$ of cases were notified by weeks 18 and 19, respectively, compared to week 21 in the two older age groups. This delay between the younger and older age groups was seen throughout all seasons but was less pronounced in seasons 2002/2003 and 2007/2008.

\section{Demographic distribution}

The mean incidence by season was $162 / 100000$ population (range 24-368) in females and 96/100000 population (range 13-228) in males. It was highest in children aged $<5$ years $(430 / 100000$ population) and in persons aged $\geqslant 75$ years $(289-1198 / 100000$ population). While among children boys were slightly more affected than girls, in the elderly a higher incidence was seen in women than in men (Fig. 3).

The cases' median age was 62 years [interquartile range (IQR) 25-82 years]. Female cases were older than male cases (median age 70 vs. 50 years, IQR 34-84 vs. 7-74 years) and occurred more often in outbreaks (72 vs. $59 \%)$.

\section{Clinical aspects}

Of cases with information (98\%), 230057 were hospitalized. For $130176(57 \%)$ of these, information on date of symptom onset and of hospitalization was available. Of these, $50 \%$ had symptom onset $\geqslant 1$ day after hospital admission and were thus considered nosocomial cases. The minimum proportion of nonnosocomial, i.e. community-acquired, hospitalized norovirus gastroenteritis in all cases was therefore $8 \%$ ( $3 \%$ in season $2003 / 2004$ to $9 \%$ in $2008 / 2009$ ). The maximum proportion was $19 \%$ (9\% in 2001/ 2002 to $23 \%$ in 2008/2009), assuming that all cases with missing information had community-acquired disease (Table 2). The proportions were $2-10 \%$ in children aged $<6$ years, and $11-24 \%$ in $\geq 75$-year-olds. 
Table 2. Characteristics of norovirus seasons 2001/2002 to 2008/2009, Germany

\begin{tabular}{|c|c|c|c|c|c|c|c|c|c|}
\hline & \multicolumn{9}{|l|}{ Season } \\
\hline & $2001 / 2002$ & $2002 / 2003$ & $2003 / 2004$ & $2004 / 2005$ & $2005 / 2006$ & 2006/2007 & 2007/2008 & $2008 / 2009$ & All \\
\hline No. of cases & 15414 & 72575 & 30501 & 93438 & 53464 & 146805 & 246800 & 197542 & 856539 \\
\hline Incidence (cases/100 000 population) & $18 \cdot 7$ & 87.9 & $37 \cdot 0$ & $113 \cdot 3$ & $64 \cdot 9$ & $178 \cdot 3$ & $300 \cdot 2$ & $240 \cdot 9$ & $130 \cdot 1$ \\
\hline Laboratory-confirmed cases (\%) & 33 & 31 & 55 & 53 & 38 & 43 & 52 & 58 & 49 \\
\hline $\begin{array}{l}\text { Minimum/maximum community-acquired cases } \\
\text { hospitalized (\%) }\end{array}$ & $4-9$ & $5-14$ & $3-10$ & $6-16$ & $5-13$ & $8-20$ & $9-22$ & $9-23$ & $8-19$ \\
\hline Deceased cases (\%) & $0 \cdot 12$ & $0 \cdot 11$ & 0.03 & 0.03 & $0 \cdot 02$ & $0 \cdot 04$ & $0 \cdot 04$ & $0 \cdot 02$ & $0 \cdot 04$ \\
\hline Female cases $(\%)$ & 66 & 71 & 63 & 64 & 65 & 65 & 63 & 61 & 64 \\
\hline Median age of cases, years (IQR) & $48(14-81)$ & $71(39-84)$ & $41(7-76)$ & $54(20-80)$ & $53(18-81)$ & $68(32-83)$ & $64(25-82)$ & $61(23-81)$ & $62(25-82)$ \\
\hline Median season week* of reporting (IQR) & $34(26-41)$ & $22(18-28)$ & $32(21-38)$ & $24(19-31)$ & $31(24-36)$ & $28(23-35)$ & $25(20-30)$ & $27(21-32)$ & $26(21-33)$ \\
\hline Cases in outbreaks (\%) & 88 & 83 & 56 & 60 & & 73 & 65 & 60 & 67 \\
\hline No. of outbreaks with sample at CL (\%) & $71(100)$ & $135(100)$ & $27(100)$ & $71(100)$ & $61(100)$ & $140(100)$ & $88(100)$ & $72(100)$ & $665(100)$ \\
\hline GI & $6(8)$ & $4(3)$ & $3(11)$ & $3(4)$ & $0(0)$ & $11(8)$ & $12(14)$ & $6(8)$ & $45(7)$ \\
\hline GII & $65(92)$ & $131(97)$ & $24(89)$ & $67(94)$ & $59(97)$ & $128(91)$ & $74(84)$ & $65(90)$ & $613(92)$ \\
\hline GI+II & $0(0)$ & $0(0)$ & $0(0)$ & $1(1)$ & $2(3)$ & $1(1)$ & $2(2)$ & $1(1)$ & $7(1)$ \\
\hline GII.4 & $43(61)$ & $127(94)$ & $9(33)$ & $60(85)$ & $16(26)$ & $104(74)$ & $58(66)$ & $50(69)$ & $467(70)$ \\
\hline
\end{tabular}

G, Genogroup; IQR, interquartile range.

Note that data above and below the horizontal line are from two different sources: the electronic reporting system and the Consultant Laboratory for Noroviruses (CL), respectively.

* Season week 1 corresponds to calendar week 31, season week 21 to calendar week 52, season week 22 to calendar week 1 (in season 2004/2005 season week 23 corresponds to calendar week 1 due to 53 calendar weeks in 2004), etc. (see Methods section). 


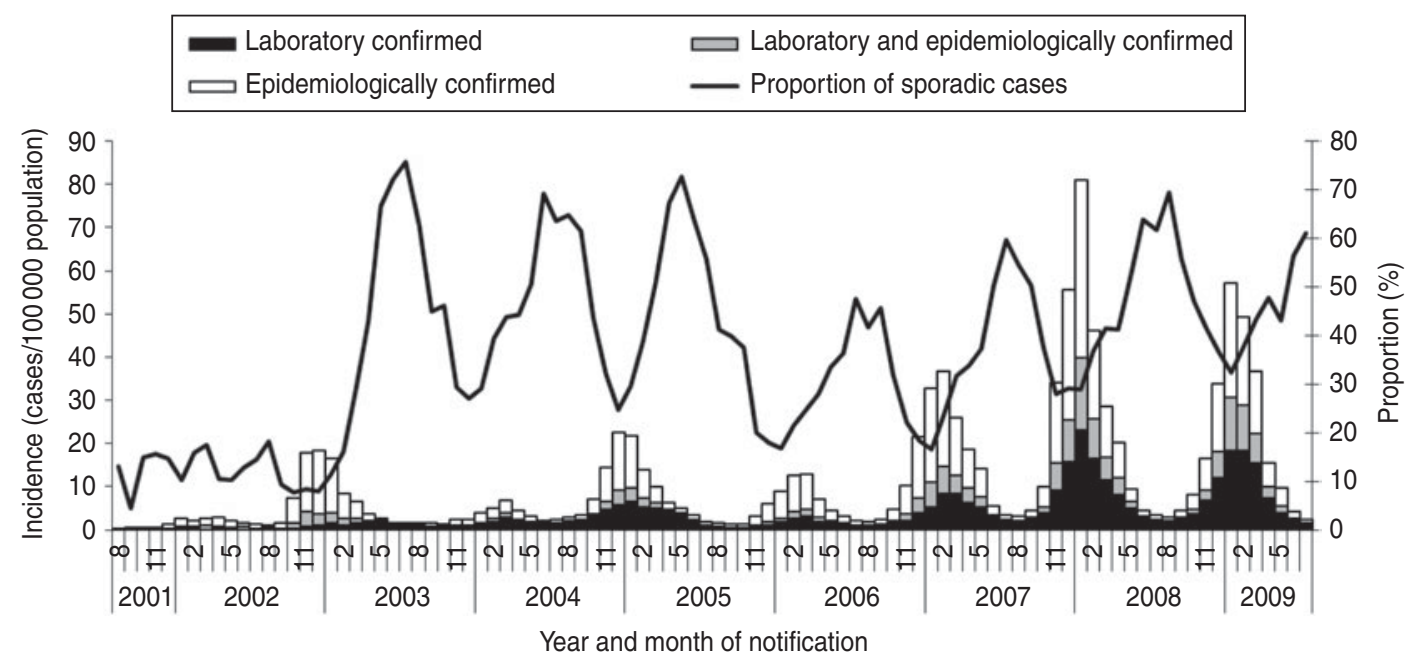

Fig. 1. Monthly incidence of reported norovirus cases by confirmation status and monthly proportion of sporadic cases, Germany, seasons 2001/2002 to 2008/2009.

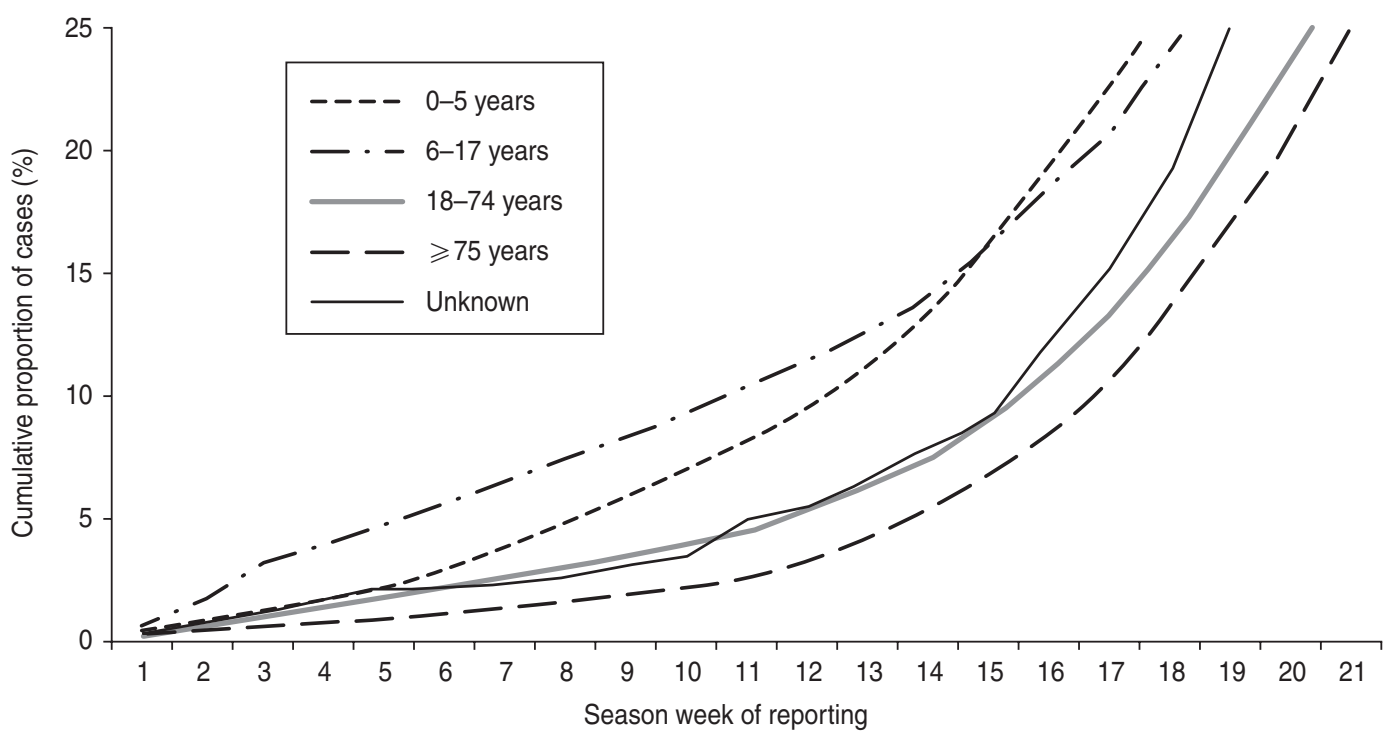

Fig. 2. Weekly cumulative proportion of notified cases by age group, Germany, seasons 2001/2002 to 2008/2009. Season week 1 corresponds to calendar week 31, season week 21 to calendar week 52 (see Methods section).

Of cases with information (99\%), a total of 326 deaths associated with norovirus gastroenteritis were reported which corresponds to a mean mortality of $0 \cdot 05 / 100000$ population by season (range $0 \cdot 01-0 \cdot 11$ ). It was comparable between females and males $(0.06$ vs. $0 \cdot 04 / 100000$, range $0 \cdot 01-0 \cdot 13$ vs. $0 \cdot 01-0 \cdot 08)$ and considerably higher $(0 \cdot 5 / 100000$, range $0 \cdot 1-1 \cdot 1)$ in persons aged $\geq 75$ years. Deceased cases had a median age of 84 years (IQR 78-90 years) compared to 62 years (IQR 24-82 years) for non-deceased cases. The proportion of deceased cases was the same $(0 \cdot 04 \%)$ in sporadic and outbreak cases.

\section{Geographical distribution}

Of the cases with information (95\%), almost all $(99 \cdot 7 \%)$ were reported to have acquired the infection in Germany. The mean incidence by season ranged from 82/100000 population in the federal state of Bavaria to $287 / 100000$ population in Saxony. It was considerably higher in the Eastern (222-287/100000 population, except for Berlin) than in the Western (82-183/100000 population) federal states. This pattern was seen throughout all seasons. 


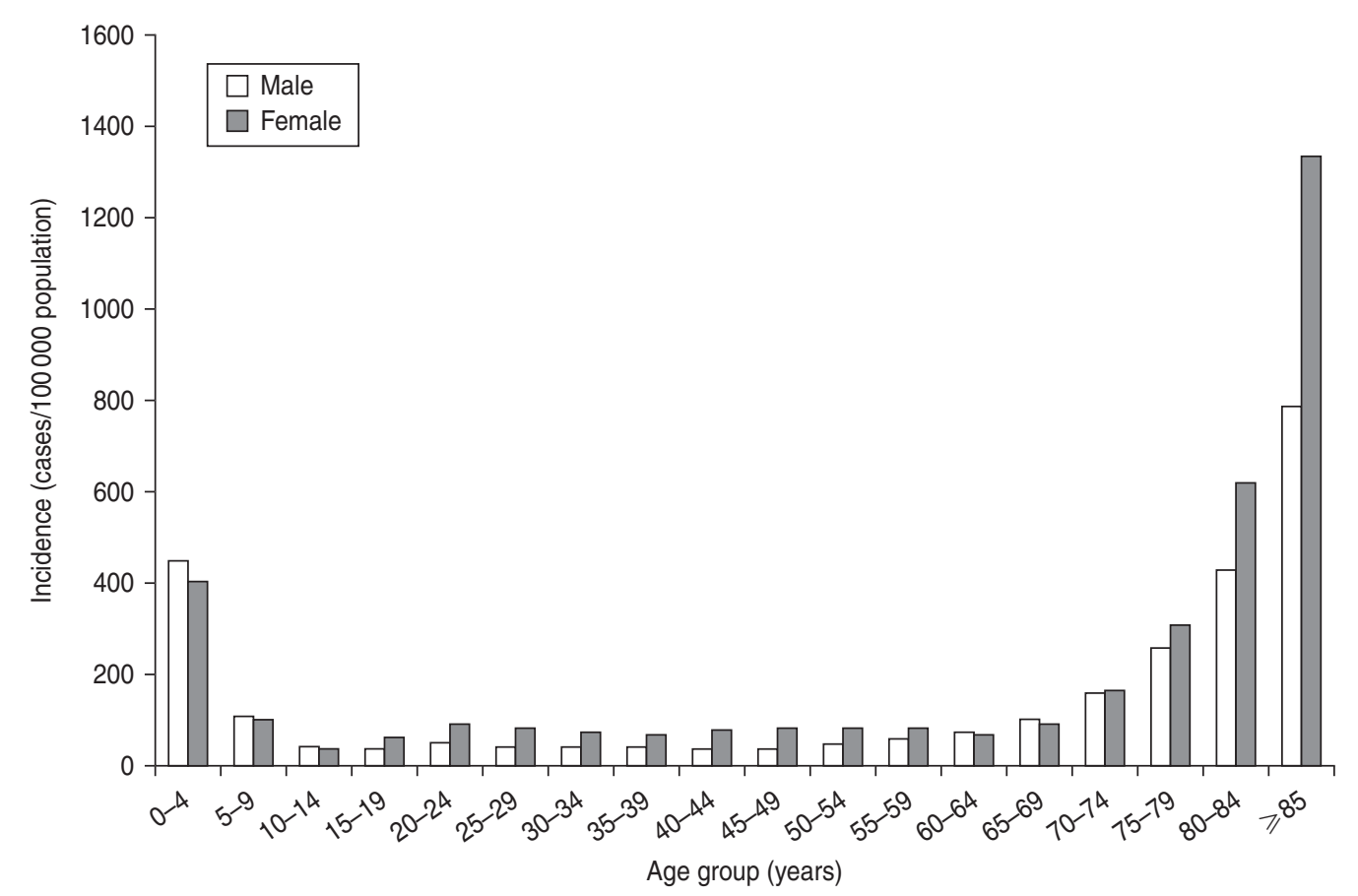

Fig. 3. Mean incidence of reported norovirus cases per season by age group and sex, Germany, seasons 2001/2002 to $2008 / 2009$.

In the East, the proportion of all hospitalized cases was lower than in the West $(20 \%$ vs. $31 \%)$ but was comparable when looking at hospitalized community-acquired illness (minimum/maximum proportions $9 \% / 12 \%$ vs. $7 \% / 23 \%$ ). The proportion of deceased cases was lower in the East $(0.01 \mathrm{vs}$. $0.05 \%$ ), as was the median age of cases (54 vs. 67 years, IQR $10-80$ vs. 31-82 years). The proportion of laboratory-confirmed cases was comparable (51\% vs. $48 \%$ ).

\section{Outbreaks}

We considered 31644 reported norovirus outbreaks for our analyses giving a total of 552823 cases. In accordance with case numbers by season, the number of outbreaks increased from 646 in season 2001/2002 to 9753 in season $2007 / 2008$ (Fig. 4).

Of the outbreaks with a reported setting (75\%), half occurred in nursing homes or hospitals followed by outbreaks in private households and childcare facilities. Outbreaks in schools or universities, residential homes (for children, adolescents, university students, soldiers), hotels, cruise ships, youth camps, and restaurants/canteens were rarely reported (Table 3).

The median case number per outbreak was nine (IQR 3-23). Outbreaks in nursing homes were larger than outbreaks in hospitals, childcare facilities or private households. Of the cases in the 31644 outbreaks, a median of two (IQR 1-5) were laboratoryconfirmed, corresponding to a median of $33 \%$ (IQR $14-63 \%$ ) of cases. The median proportion of laboratory-confirmed cases depended on the outbreak setting and was low in nursing homes (14\%) and childcare facilities (17\%), higher in hotel outbreaks $(35 \%)$ and highest in private households, restaurants/ canteens and hospitals $(50 \%)$. The median age of cases per outbreak was 67 years (IQR 24-80 years) and reflected the expected age of persons represented in the respective settings. The proportion of female cases was high $(80 \%)$ in nursing home outbreaks, whereas the sex distribution was more comparable in all other settings (Table 3 ).

Similar to the results from case data, outbreaks in childcare facilities and schools occurred 5-6 weeks earlier in the norovirus season than outbreaks in other settings with sufficiently high outbreak numbers during the study period, i.e. in nursing homes, hospitals and private households (Table 3). This trend was seen throughout all seasons except for seasons 2001/2002 and 2002/2003, during which only one outbreak in a childcare facility was reported.

For $799(3 \%)$ outbreaks a link to a specific food item was reported. The proportion varied between 


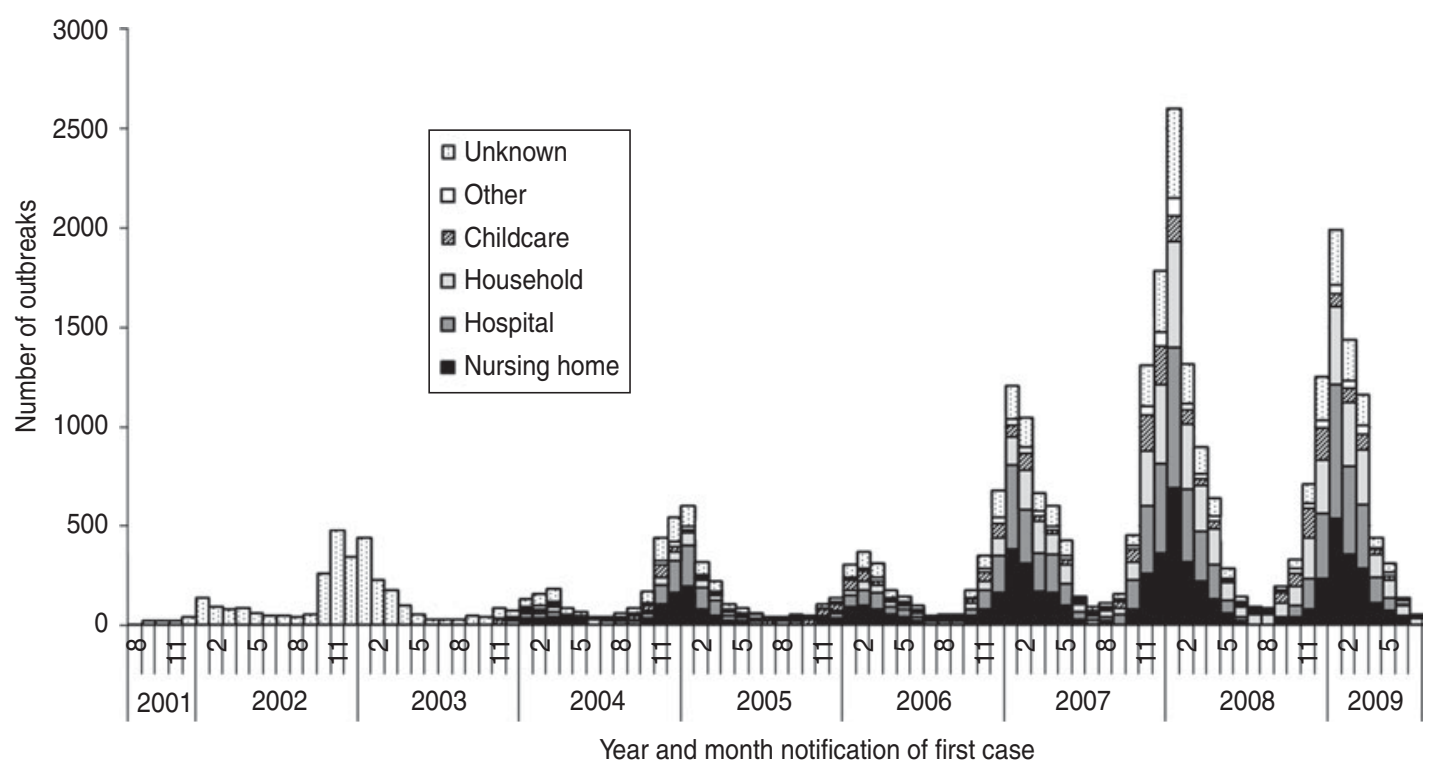

Fig. 4. Distribution of settings of reported norovirus outbreaks $(n=31644)$ by year and month of reporting, Germany, seasons 2001/2002 to 2008/2009.

the outbreak setting and was highest for restaurant and canteen outbreaks $(46 \%, n=66)$ and for outbreaks in hotels, on cruise ships or in youth camps $(9 \%$, $n=19$ ).

\section{Molecular characteristics of outbreaks}

From August 2001 to July 2009, a total of 996 clinical specimens from 665 norovirus outbreaks were genotyped at the CL. In $92 \%$ of the outbreaks, norovirus genogroup GII was identified, ranging from $84 \%$ in season $2007 / 2008$ to $97 \%$ in seasons $2002 / 2003$ and 2005/2006 (Table 2). GI strains were identified in $7 \%$ of all outbreaks, and in $1 \%$ of outbreaks both genogroups were found.

GII strains dominated outbreaks in all reported settings, but GI strains were found in a higher proportion of outbreaks in childcare facilities, schools, hotels, cruise ships and youth camps than in other settings (Table 3). Whereas GI strains were found in outbreaks occurring all year round, i.e. also during summer, GII strains showed a stronger seasonality and were more often found during winter (Fig. 5a).

Most GII outbreaks were associated with genotype GII.4 (76\%) followed by GII.7 (10\%), recombinant GII.b/GII.3 (7\%), and GII.2 (3\%). The highest proportion of outbreaks with GII.4 was found in season $2002 / 2003(94 \%$, Table 2), compared to only $26 \%$ in season 2005/2006 when genotype GII.7 (51\%) dominated. In 2003/2004 recombinant GII.b/GII.3 (42\%) was the most frequently detected strain (Fig. 5a). The proportion of GII.4 outbreaks was $82 \%$ $(174 / 211)$ in nursing homes and 85\% (203/239) in hospitals, but only $33 \%(45 / 138)$ in childcare facilities and schools (Table 3).

Starting from 2002, several GII.4 variants emerged in Germany, became predominant and were replaced by the next emerging variant (Fig. $5 b$ ).

\section{DISCUSSION}

We have described data on over 850000 cases and 31000 outbreaks from eight seasons of norovirus surveillance in Germany. Our data include detailed information on laboratory and epidemiologically confirmed cases. This has been a unique feature of the German norovirus surveillance system from 2001 to 2009 compared to other countries where surveillance focuses on outbreak surveillance or laboratory reports $[14,15]$.

Our incidences per season are lower than estimates of 4500-6500 cases/100000 person-years from the USA and UK [16-18] and of 3800 cases/100000 population from The Netherlands [19], and also lower than 1665 cases $/ 100000$ children aged $<5$ years in industrialized countries from a systematic literature review on sporadic gastroenteritis [2]. This is plausible because our passive surveillance system probably underestimates the true incidence of norovirus disease in Germany, especially in the young and healthy 
Table 3. Characteristics of reported norovirus outbreaks with known setting ( $n=23788$ ) by outbreak setting, seasons 2001/2002 to 2008/2009, Germany

\begin{tabular}{|c|c|c|c|c|c|c|c|c|c|}
\hline & \multicolumn{9}{|c|}{ Outbreak setting } \\
\hline & $\begin{array}{l}\text { Nursing } \\
\text { home }\end{array}$ & Hospital & $\begin{array}{l}\text { Childcare } \\
\text { facility }\end{array}$ & $\begin{array}{l}\text { Private } \\
\text { household }\end{array}$ & $\begin{array}{l}\text { School, } \\
\text { university }\end{array}$ & $\begin{array}{l}\text { Residential } \\
\text { home }\end{array}$ & $\begin{array}{l}\text { Hotel, } \\
\text { cruise ship, } \\
\text { youth camp }\end{array}$ & $\begin{array}{l}\text { Restaurant, } \\
\text { canteen }\end{array}$ & Other \\
\hline No. of outbreaks (\%) & $6768(28)$ & $7674(32)$ & $2384(10)$ & $5730(24)$ & $148(<1)$ & $314(1)$ & $223(<1)$ & $145(<1)$ & $402(2)$ \\
\hline Median case no. (IQR) & $22(11-38)$ & $10(5-21)$ & $13(8-20)$ & $3(2-4)$ & $20(10-35)$ & $12(7-20)$ & $5(2-15)$ & $6(3-14)$ & $7(3-16)$ \\
\hline $\begin{array}{l}\text { Median no of laboratory-confirmed } \\
\text { cases (IQR) }\end{array}$ & $3(2-5)$ & $4(2-9)$ & $2(1-3)$ & $1(1-2)$ & $2(1-4)$ & $2(1-4)$ & $2(1-3)$ & $3(2-5)$ & $2(1-4)$ \\
\hline Median $\%$ cases female (IQR) & $80(70-87)$ & $62(50-75)$ & $50(42-63)$ & $50(33-67)$ & $50(40-59)$ & $57(43-71)$ & $50(43-67)$ & $50(33-67)$ & $50(33-67)$ \\
\hline Median age of cases, years (IQR) & $82(77-84 \cdot 5)$ & $73(62-79)$ & $4(2-4 \cdot 5)$ & $25(14-38)$ & $10(8-16 \cdot 25)$ & $43 \cdot 5(29-54)$ & $29 \cdot 5(15-58)$ & $41(32-47 \cdot 5)$ & $37 \cdot 5(21-52)$ \\
\hline Foodborne outbreaks (\%) & 3 & 2 & 2 & 5 & 7 & 3 & 9 & 46 & 9 \\
\hline $\begin{array}{l}\text { Median season week* of outbreak } \\
\text { start (IQR) }\end{array}$ & $26(22-32)$ & $26(21-32)$ & $21(17-29)$ & $27(20-33)$ & $21(18-29 \cdot 5)$ & $27(21-33)$ & $27(13-43)$ & $24(19-38)$ & $26(19-35)$ \\
\hline $\begin{array}{l}\text { No. of outbreaks with sample } \\
\text { at CL ( } \% \text { of total) }\end{array}$ & $211(100)$ & $239(100)$ & $138(100)$ & $8(100)$ & $\dagger$ & $t$ & $6(100)$ & $5(100)$ & $56(100)$ \\
\hline GI & $12(6)$ & $4(2)$ & $18(13)$ & $0(0)$ & & & $1(17)$ & $0(0)$ & $8(14)$ \\
\hline GII & $197(93)$ & $233(97)$ & $119(86)$ & $8(100)$ & & & $5(83)$ & $5(100)$ & $46(82)$ \\
\hline $\mathrm{GI}+\mathrm{II}$ & $2(1)$ & $2(1)$ & $1(1)$ & $0(0)$ & & & $0(0)$ & $0(0)$ & $2(4)$ \\
\hline GII.4 & $174(82)$ & $203(85)$ & $45(33)$ & $4(50)$ & & & $4(67)$ & $2(40)$ & $35(63)$ \\
\hline
\end{tabular}

G, Genogroup; IQR, interquartile range.

Note that data above and below the horizontal line are from two different sources: the electronic reporting system and the Consultant Laboratory for Noroviruses (CL), respectively.

* Season week 1 corresponds to calendar week 31, season week 21 to calendar week 52, season week 22 to calendar week 1 (in season $2004 / 2005$ season week 23 corresponds to calendar week 1 due to 53 calendar weeks in 2004), etc. (see Methods section).

† School outbreaks included in 'childcare facility'.

$\$$ Residential home outbreaks included in 'nursing home'. 


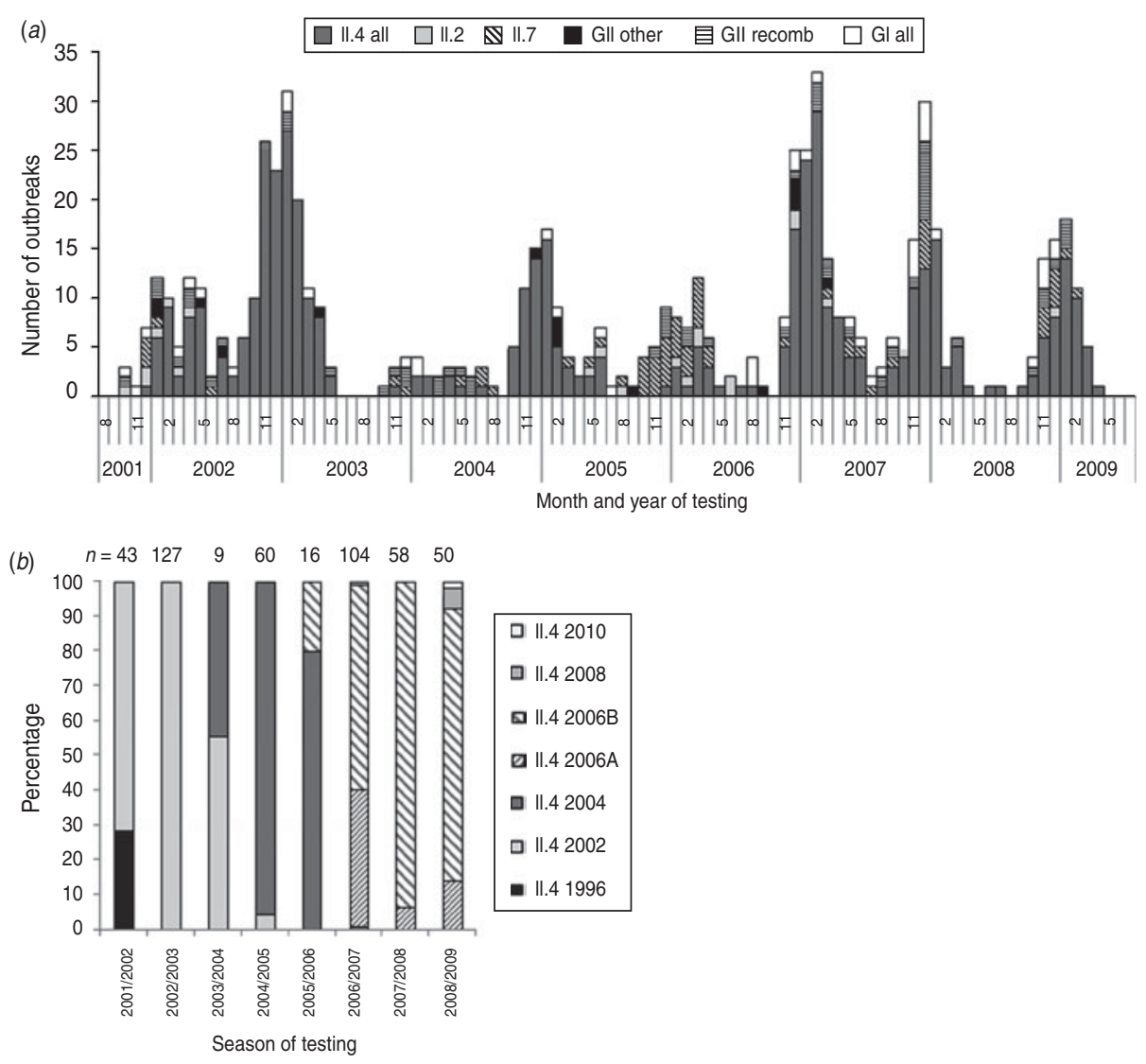

Fig. 5. (a) Monthly distribution of norovirus genotypes, August 2001-July 2009 and (b) distribution of genotype II.4 variants in seasons 2001/2002 to 2008/2009 in outbreaks with samples analysed at the Consultant Laboratory for Noroviruses (CL). n, Total number of samples at CL; GII other=II.5, II.8, II.12, II.14, II.20, GII/GI mix; GII recomb= II.b, II.d, II.e, II.g.

population, who do not consult the healthcare system for uncomplicated acute gastroenteritis.

The proportion of hospitalized cases of communityacquired illness was $8-19 \%$. The true proportion, which we assumed to be around $13 \%(6 \%$ in $<6$ yearolds, $17 \%$ in $>74$-year-olds), is considerably higher than the $0.7 \%$ reported in a review of published outbreaks which excluded hospital outbreaks [20] and the $0.03 \%$ estimated for foodborne norovirus illness [21]. Moreover, the proportion in <6-year-olds is lower than the $12 \%$ reported for children aged $<5$ years with sporadic gastroenteritis [2]. However, the population underlying published outbreak reports differs substantially from the population covered by our routine surveillance, and only a small percentage of our outbreaks were reported to be foodborne; therefore, comparability with results from the abovementioned publications is limited. It is unlikely that norovirus cases are hospitalized more often in Germany than in other countries. It is more likely that our surveillance system under-ascertained uncomplicated community-acquired cases which escape the attention of the healthcare system leading to a higher proportion of hospitalized cases. The increasing proportion of hospitalized cases over time can be explained by the increasing number of outbreak reports from nursing homes where the residents are more likely to be prone to more severe courses of disease. Additionally, in this setting awareness and testing for norovirus is probably increased.

The relatively constant proportion of fatal cases over time (mean: $0 \cdot 04 \%$ ) also supports the notion that norovirus disease severity is not increasing over time. Our results are comparable to the $0.03 \%$ in longterm care facilities in Australia [22] and the 0.07\% described in a review of published outbreaks [20], considering that our dataset includes sporadic cases that are younger and less likely to develop complications. Our mortality rate of $0 \cdot 5 / 100000$ cases per season in the elderly ( $\geqslant 75$ years) is lower than modelling estimates from the USA, UK and The Netherlands of $2 / 100000,0 \cdot 6 / 100000$ and $3 / 100000$, respectively 
[3, 23, 24], but comparable when considering that the surveillance system captures only half of norovirusassociated deaths compared to the German causeof-death statistics [25].

We found that norovirus incidence and mortality were higher in females than in males, and female cases were older than male cases. These findings can be explained by the higher proportion of females in nursing homes, where the risk of infection during the norovirus high season as well as the likelihood of outbreaks being recognized might be higher than in other settings.

The incidence in the Eastern German federal states was higher than in the Western states. Considering the findings that cases in the East are younger (especially sporadic laboratory-confirmed cases, data not shown) and the proportion of deceased cases is lower, we believe that the higher reporting incidences reflect a different behaviour regarding medical consultation and laboratory testing rather than a true difference in incidence.

The increase in case numbers from 2001/2002 to $2006 / 2007$ has also been reported in other countries and is in part probably due to the increased worldwide circulation of GII.4 strains [26] resulting in synchronized norovirus epidemics in Europe, USA, and Canada [7]. GII.4 strains have been reported to cause more severe illness $[20,27]$ and a higher viral load in infected persons than other strains [28], which may lead to more frequent disease transmission. A higher environmental stability has also been discussed [26]. However, increased awareness for norovirus entailing more laboratory testing, and the availability of more sensitive tests such as real-time RT-PCR [1] have probably also contributed to increasing case numbers in the early 2000s. The increasing proportion of laboratory-confirmed cases over the eight seasons could support this notion, but from anecdotal reports we attribute this to an underascertainment of epidemiologically confirmed cases in the context of large norovirus epidemics implying a high workload.

Interestingly, we found that the norovirus season started earlier in the younger $(<18$ years) than in the older age groups which was supported by case-based as well as outbreak data. Whether this reflects a pattern of disease transmission from young to old in the community should be investigated in further studies. One outbreak investigation from Sweden found children to be more likely to cause secondary cases [29]. Whether this is linked to the longer symptomatic period [30] or the higher frequency of vomiting in children [31] is unclear. It is subject to controversy whether vomiting poses a higher risk of norovirus transmission than other routes of transmission [32]. An alternative explanation for the observed antecedence of children each season could be that the strains causing disease in the younger age groups differ from those in adults implying that epidemiological patterns in children and adults are not necessarily related to each other. In our convenience sample, outbreaks in childcare settings were less frequently caused by GII.4 (and more frequently by GI) strains than hospital and nursing home outbreaks, which corresponds to a recent publication that reported a higher frequency of GII.4 strains in outbreaks in healthcare settings than in outbreaks involving younger persons [5]. Findings from France suggest that GII noroviruses are more common in sporadic cases whereas GI strains are predominant in outbreaks in children [33].

The distribution of our outbreak settings differed from that in other countries [14, 26, 34, 35]. One probable reason is that our dataset includes a large number of outbreaks in private households which are not captured as frequently by other surveillance systems. This might also explain why, in contrast to outbreak surveillance data from England and Wales [34], we found a seasonal variation with a winter peak for outbreaks in all settings.

The number of foodborne outbreaks in our database is unclear. According to a recent publication [21] $26 \%$ of norovirus illnesses in the USA are foodborne. In Germany, the LPHD can report an implicated vehicle of infection for each outbreak, but often no investigation is performed on whether a norovirus outbreak is primarily foodborne if the setting (e.g. restaurant) does not imply this. Even in restaurant and canteen outbreaks only $47 \%$ were reported to be foodborne. However, the proportion of foodborne outbreaks by outbreak setting in our dataset roughly resembles data from England and Wales [34]. We probably underestimated the amount of foodborne norovirus transmission but cannot quantify it from our data.

There are some limitations to our findings. Routine surveillance data do not provide the complete picture of norovirus activity in the population. In particular, uncomplicated cases and cases without laboratory confirmation are likely to be under-represented in our database. We thus underestimated the size of large outbreaks, e.g. in hospitals and nursing homes [36], but more importantly we under-ascertained 
small outbreaks in private households and sporadic community cases as has been shown for the UK [18]. However, the ratio of median outbreak size by setting reflects what we expected to find according to the size of the population at risk in the respective settings and is comparable to outbreak surveillance data from Australia [22]. We therefore believe that our surveillance data reflect the correct trend of norovirus activity in the different settings.

A major limitation is that our typing data on outbreaks come from a convenience sample. We must assume that this sample might not be representative for outbreaks in Germany, not only because the distribution of outbreak settings in the laboratory data (household outbreaks were basically not represented) differed considerably from that in the routine surveillance data. From our experience, diagnostic laboratories, physicians and local public health authorities send outbreak specimens to the CL not only to ensure certain diagnostic standards, but also when the outbreak at hand is of special media interest or when the genogroup is difficult to identify. Therefore outbreaks that are exceptional in regard to the norovirus strain involved, disease severity or size are probably over-represented in the dataset.

In conclusion, our routine surveillance data on norovirus disease in Germany confirm estimates on norovirus disease burden from other countries. We found some evidence for a start of the norovirus season in younger age groups. More detailed investigation is required in the future on transmission patterns between children and adults in order to identify possible starting points for enhanced prevention of disease spread in the population.

\section{ACKNOWLEDGEMENTS}

We are indebted to the staff of the local public health authorities who dedicated their time and effort to collect data on cases and outbreaks since 2001. We also thank Mirko Faber, Michael Höhle and Dirk Werber for helpful discussions on the surveillance data. This research received no specific grant from any funding agency, commercial or not-for-profit sectors.

\section{DECLARATION OF INTEREST}

None.

\section{REFERENCES}

1. Glass RI, Parashar UD, Estes MK. Norovirus gastroenteritis. New England Journal of Medicine 2009; 361: 1776-1785.

2. Patel MM, et al. Systematic literature review of role of noroviruses in sporadic gastroenteritis. Emerging Infectious Diseases 2008; 14: 1224-31.

3. Harris JP, et al. Deaths from norovirus among the elderly, England and Wales. Emerging Infectious Diseases 2008; 14: 1546-1552.

4. Roddie C, et al. Allogeneic hematopoietic stem cell transplantation and norovirus gastroenteritis: a previously unrecognized cause of morbidity. Clinical Infectious Diseases 2009; 49: 1061-1068.

5. Matthews JE, et al. The epidemiology of published norovirus outbreaks: a review of risk factors associated with attack rate and genogroup. Epidemiology and Infection 2012; 140: 1161-1172.

6. Zheng DP, et al. Norovirus classification and proposed strain nomenclature. Virology 2006; 346: 312-23.

7. Siebenga JJ, et al. Norovirus illness is a global problem: emergence and spread of norovirus GII.4 variants, 2001-2007. Journal of Infectious Diseases 2009; 200: $802-812$.

8. Bok K, et al. Evolutionary dynamics of GII.4 noroviruses over a 34-year period. Journal of Virology 2009; 83: 11890-901.

9. Robert Koch Institute. Case definitions of the RKI for transmission of illnesses or deaths and of detection of infectious disease pathogens [in German]. Berlin (Germany): Robert Koch Institute, 2007.

10. Vinje J, Hamidjaja RA, Sobsey MD. Development and application of a capsid VP1 (region D) based reverse transcription PCR assay for genotyping of genogroup I and II noroviruses. Journal of Virological Methods 2004; 116: 109-117.

11. Hoehne M, Schreier E. Detection of Norovirus genogroup I and II by multiplex real-time RT-PCR using a 3'-minor groove binder-DNA probe. BMC Infectious Diseases 2006; 6: 69.

12. Oh DY, Gaedicke G, Schreier E. Viral agents of acute gastroenteritis in German children: prevalence and molecular diversity. Journal of Medical Virology 2003; 71: 82-93.

13. Felsenstein J. PHYLIP - Phylogeny interference package (Version 3.2). Cladistics 1989; 5: 164-166.

14. Siebenga JJ, et al. Gastroenteritis caused by norovirus GGII.4, The Netherlands, 1994-2005. Emerging Infectious Diseases 2007; 13: 144-146.

15. Wall PG, et al. Food poisoning: notifications, laboratory reports, and outbreaks - where do the statistics come from and what do they mean? Communicable Disease Report. CDR Review 1996; 6: R93-100.

16. Hall AJ, et al. Incidence of acute gastroenteritis and role of norovirus, Georgia, USA, 2004-2005. Emerging Infectious Diseases 2011; 17: 1381-1388.

17. Phillips G, et al. Community incidence of norovirusassociated infectious intestinal disease in England: improved estimates using viral load for norovirus 
diagnosis. American Journal of Epidemiology 2010; 171: 1014-1022.

18. Tam CC, et al. Longitudinal study of infectious intestinal disease in the UK (IID2 study): incidence in the community and presenting to general practice. Gut 2012; 61: 69-77.

19. Verhoef L, et al. The estimated disease burden of norovirus in The Netherlands. Epidemiology and Infection. Published online: 17 May 2012. doi:10.1017/ S0950268812000799.

20. Desai R, et al. Severe outcomes are associated with genogroup 2 genotype 4 norovirus outbreaks: a systematic literature review. Clinical Infectious Diseases 2012; 10.1093/cid/cis372.

21. Scallan E, et al. Foodborne illness acquired in the United States-major pathogens. Emerging Infectious Diseases 2011; 17: 7-15.

22. Kirk MD, et al. Surveillance for outbreaks of gastroenteritis in long-term care facilities, Australia, 20022008. Clinical Infectious Diseases 2010; 51: 907-914.

23. Hall AJ, et al. The roles of Clostridium difficile and norovirus among gastroenteritis-associated deaths in the United States, 1999-2007. Clinical Infectious Diseases 2012; 55: 216-223.

24. van Asten $\mathbf{L}$, et al. Unspecified gastroenteritis illness and deaths in the elderly associated with norovirus epidemics. Epidemiology 2011; 22: 336-343.

25. Werber D, et al. Years of potential life lost for six major enteric pathogens, Germany, 2004-2008. Epidemiology and Infection. Published online: 20 July 2012. doi:10.1017/S0950268812001550.

26. Lopman B, et al. Increase in viral gastroenteritis outbreaks in Europe and epidemic spread of new norovirus variant. Lancet 2004; 363: 682-688.

27. Huhti L, et al. Norovirus GII-4 causes a more severe gastroenteritis than other noroviruses in young children. Journal of Infectious Diseases 2011; 203: 1442-1444.

28. Chan MC, et al. Fecal viral load and norovirusassociated gastroenteritis. Emerging Infectious Diseases 2006; 12: 1278-1280.

29. Gotz H, et al. Clinical spectrum and transmission characteristics of infection with Norwalk-like virus: findings from a large community outbreak in Sweden. Clinical Infectious Diseases 2001; 33: 622-628.

30. Rockx B, et al. Natural history of human calicivirus infection: a prospective cohort study. Clinical Infectious Diseases 2002; 35: 246-253.

31. Arias C, et al. Epidemiological and clinical features of norovirus gastroenteritis in outbreaks: a populationbased study. Clinical Microbiology and Infection 2010; 16: $39-44$.

32. Sukhrie FH, et al. Nosocomial transmission of norovirus is mainly caused by symptomatic cases. Clinical Infectious Diseases 2012; 54: 931-937.

33. Bon F, et al. Molecular epidemiology of caliciviruses detected in sporadic and outbreak cases of gastroenteritis in France from December 1998 to February 2004. Journal of Clinical Microbiology 2005; 43: 4659-4664.

34. Lopman BA, et al. Two epidemiologic patterns of norovirus outbreaks: surveillance in England and wales, 1992-2000. Emerging Infectious Diseases 2003; 9: $71-77$.

35. Blanton LH, et al. Molecular and epidemiologic trends of caliciviruses associated with outbreaks of acute gastroenteritis in the United States, 2000-2004. Journal of Infectious Diseases 2006; 193: 413-421.

36. Hauri AM, et al. Electronic outbreak surveillance in Germany: a first evaluation for nosocomial norovirus outbreaks. PLoS One 2011; 6: e17341. 